\title{
Vectorial Chaos Synchronization and Polarization Encoding in Self-Pulsating VCSELs
}

\author{
Alessandro Scirè ${ }^{* a}$, Josep Mulet ${ }^{a}$, Claudio R. Mirasso ${ }^{b}$, Jan Danckaert $^{c, a}$ and Maxi San Miguel ${ }^{a}$ \\ ${ }^{a}$ Instituto Mediterráneo de Estudios Avanzados (IMEDEA), \\ Consejo Superior de Investigaciones Científicas-Universitat de les Illes Balears, \\ Campus Universitat Illes Balears, E-07071 Palma de Mallorca, Spain. \\ ${ }^{b}$ Departament de Física, Universitat de les Illes Balears. E-07071 Palma de Mallorca, Spain. \\ ${ }^{c}$ Vrije Universiteit Brussel, Dept. of Applied Physics and Photonics (TW - TONA) \\ Pleinlaan 2, B-1050 Brussels, Belgium.
}

\begin{abstract}
In this contribution, we analyze the synchronization properties of two chaotic optical field generated by two VCSELs in presence of a saturable absorber. The vectorial nature of the chaotic field requires a continuous control scheme to achieve a high-quality synchronization between the two sources. We proposed a novel encoding scheme, exploiting a two contact VCSEL structure, which allows an easy and direct modulation of the transmitter. The added degree of freedom constituted by the chaos in polarization allows a reduction of the probability error (or enhancement of the transmission capacity) at the receiver.
\end{abstract}

\section{INTRODUCTION}

The synchronization of chaotic nonlinear oscillators has attracted much attention in recent years, motivated by the possibility of practical applications of this fundamental phenomenon. Several papers ${ }^{1}$ have shown that such synchronization may be achieved in electronic oscillator circuits, with application in the transmission of information signals masked in a background of chaos. While assessing the synchronization techniques and the theoretical understanding of those phenomena, many optical systems were also shown to exhibit chaos in the past decade. Therefore many concepts and ideas concerning the synchronization of chaotic systems have been demonstrated in optoelectronic systems, first theoretically ${ }^{2}$ and then experimentally. ${ }^{3}$ The enormous success of the optical telecommunications strongly motivated the study of chaotic lasers in view of cryptographic applications. However, the synchronization scheme proposed by Pecora and $\mathrm{Carrol}^{4}$ requires the extraction of a stable subsystem (only negative Lyapunov exponents) from an existing chaotic system. When a chaotic system and a stable response subsystem are linked with a common driving signal, the two may display synchronized chaos. While this scheme has been successfully implemented with electronic oscillators, it has been found impossible to separate the elements of an optical chaotic system (e.g. a chaotic laser) to obtain a stable subsystem in precisely this manner. However, if the system is not too chaotic (i.e. the system has only one positive Lyapunov exponent), simple alternative methods can induce synchronization. One example is the synchronization achieved through direct coupling, unidirectional or mutual, between two semiconductor chaotic lasers, which has been proved both theoretically and experimentally. ${ }^{5-7}$ Semiconductor lasers have been widely exploited in this context, due to their high nonlinearity, low cost and wide diffusion. Among these devices, Vertical-Cavity Surface-Emitting Lasers (VCSELs) ${ }^{8}$ are important compact light sources for applications, e.g. in optical interconnects and optical data storage, due to their low threshold current, single-longitudinal mode operation and easy integrability in 2-D arrays. Recently, a new source exhibitng optical chaos, based on a VCSEL with a surrounding saturable absorber, has been investigated. ${ }^{9,10}$ This source can emit in a chaotic state of both total intensity and polarization (we call it vectorial chaos), thus seeming promising for widening the knowledge in the chaotic lasers and enhancing the transmission capacity in view of cryptographic applications.

In this paper, we first analyze the synchronization properties of two identical chaotic sources consisting in a VCSEL with a surrounding saturable absorber. In agreement with theoretical arguments, ${ }^{14}$ and since our system

*Other author information: (Sent correspondence to M.S.M.): Email: maxi@imedea.uib.es; Telephone: +34-971-173229; Fax: +34-971-173426; URL: http//www.imedea.uib.es 
shows at least two positive exponents (due to the total intensity and polarization instabilities), a continuous control scheme $(\mathrm{CCS})^{15}$ is found to give the best synchronization. The robustness of this scheme against parameters mismatch and noise has been investigated, with promising results in Edge Emitter Lasers (EELs) based systems. ${ }^{11,12}$ Once the synchronization is achieved, we theoretically investigate a novel scheme for message encoding based on a doublecontact VCSEL structure (analogous of the well-known two contacts EELs). In this scheme the information is added in the system through the direct modulation of the current difference between the two sections, while keeping constant the total current, thus leaving the chaotic evolution of the total intensity almost unperturbed. A decoding scheme, based on the CSK scheme ${ }^{11,12,16}$ allows to recover the information in two channels (one per polarization state), lowering the error probability. The paper is organized as follows: in Sec. 2 we introduce a model to describe a VCSEL in presence of a saturable absorber, and characterize the chaotic attractor emerging in a region of the parameter space. Subsequently, we discuss the synchronization properties of two identical systems through a CCS. In Sec. 3 we illustrate an encoding scheme based on a double-contact self-pulsating VCSEL and finally Sec. 4 is devoted to conclude and summarize our paper.

\section{MODEL AND SYNCHRONIZATION SCHEME}

The basic system consists in a VCSEL with a surrounding saturable absorption region. In order to synchronize two of these systems we propose the CCS shown in Fig. 1. The feedback loop is characterized by a response function $H(\omega)$, that, in general, can be written as ${ }^{13}$

$$
H(\omega)=\frac{H_{0} \exp (i \phi)}{1+i \omega \tau}
$$

where $H_{0}$ is the overall attenuation and $\phi$ is the dephasing, both acquired in the feedback path. The frequency response of the feedback path is assumed to be one-pole kind, with $3 d B$ attenuation frequency equal to $1 / \tau$. Previous studies $^{11}$ showed the role played by the attenuation $H_{0}$ and the frequency-cut $1 / \tau$ is normally negligible. In the rest of the paper we set $H_{0}=1$ and $\tau \rightarrow \infty$.

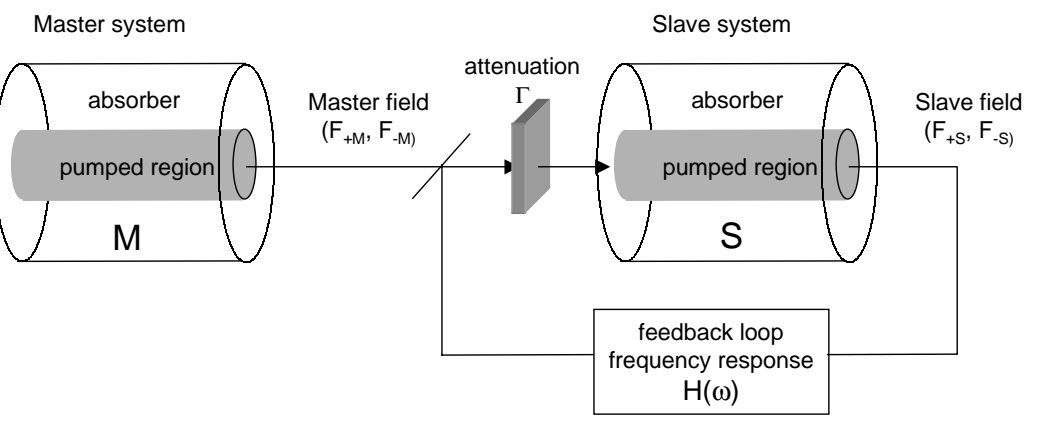

Figure 1. Continuous control synchronization scheme.

The scheme of Fig. 1 has been analyzed through numerical simulations, as detailed below. We describe the systems $M$ and $S$ with a recently developed model,, 10 suitable to describe a VCSEL in presence of a surrounding saturable absorber. The rate equations describing the dynamic evolution of the $S$ and $M$ systems read

$$
\begin{aligned}
\dot{F}_{ \pm S, M} & =\frac{1}{2}(1+i \alpha)\left[D_{1 S, M}+D_{2 S, M} \pm d_{1 S, M} \pm d_{2 S, M}-1\right] F_{ \pm S, M}-\left(\varepsilon_{a}+i \varepsilon_{p}\right) F_{\mp S, M}+f_{ \pm S, M}(t)+\Theta_{S, M},(2) \\
\dot{D}_{1 S, M} & =\gamma_{1}\left[\mu_{1}-D_{1 S, M}-\left(D_{1 S, M}+d_{1 S, M}\right)\left|F_{+S, M}\right|^{2}-\left(D_{1 S, M}-d_{1 S, M}\right)\left|F_{-S, M}\right|^{2}+c_{12} D_{2 S, M}\right] \\
\dot{D}_{2 S, M} & =\gamma_{2}\left[\mu_{2}-D_{2 S, M}-a\left(D_{2 S, M}+d_{2 S, M}\right)\left|F_{+S, M}\right|^{2}-a\left(D_{2 S, M}-d_{2 S, M}\right)\left|F_{-S, M}\right|^{2}+c_{21} D_{1 S, M}\right] \\
\dot{d}_{1 S, M} & =-\gamma_{s 1} d_{1 S, M}-\gamma_{1}\left[\left(D_{1 S, M}+d_{1 S, M}\right)\left|F_{+S, M}\right|^{2}-\left(D_{1 S, M}-d_{1 S, M}\right)\left|F_{-S, M}\right|^{2}-c_{12} d_{2 S, M}\right] \\
\dot{d}_{2 S, M} & =-\gamma_{s 2} d_{2 S, M}-\gamma_{2}\left[a\left(D_{2 S, M}+d_{2 S, M}\right)\left|F_{+S, M}\right|^{2}-a\left(D_{2 S, M}-d_{2 S, M}\right)\left|F_{-S, M}\right|^{2}-c_{21} d_{1 S, M}\right]
\end{aligned}
$$


where the subindexes $M$ and $S$ indicate the $M$ aster and $S$ lave systems, while 1 and 2 stand for the pumped and absorbing regions, of each system, respectively. $F_{ \pm M, S}$ are the slowly-varying complex amplitudes of the two circularly-polarized components of the vectorial electric field $\vec{F}_{M, S}, D_{i M, S}(i=1,2)$ are the total carrier inversion between the conduction and valence bands referred to the transparency carrier density, while $d_{i M, S}$ are the differences of the carrier inversion with opposite spin orientations. The equations are written in a dimensionless form such that the time is measured in $\kappa^{-1}$ units, being $\kappa$ the cavity decay rate. The effective injection currents, with respect to transparency, are $\mu_{1}$ and $\mu_{2}$, since the current is only supplied in the central region, $\mu_{1}>0$ while $\mu_{2}<0$. Carrier diffusion is introduced in the rate equations through the coupling terms $c_{12} D_{1 M, S}$ and $c_{21} D_{2 M, S}$. The linewidth enhancement factor $\alpha$ describes the phase amplitude coupling. The parameters $\gamma_{i}$ stand for the total carrier decay rates, while $\gamma_{s i}$ are the effective spin flip rates. The phase and amplitude anisotropies ${ }^{17}$ are $\varepsilon_{p}$ and $\varepsilon_{a}$ respectively. Since our results have been found only weakly dependent on the amplitute anisotropies, we set $\varepsilon_{a}=0$ in the rest of the paper. In order to maintain the notations of previous papers, ${ }^{17}$ we define the birefringence as $\gamma_{p}=\kappa \varepsilon_{p}$. The differential gains in the active and the absorber regions differ due to their different carrier densities. This effect is taken into account through the parameters $a$, which represents the ratio of the the effective gain coefficient in the absorbing region with respect to the pumped zone. The effective gain coefficient is calculated ${ }^{18}$ as the product of the differential gain times the fraction of optical power within each region. The Langevin noise sources ${ }^{19} f_{ \pm S, M}(t)$ can be approximated by

$$
f_{ \pm S, M}(t)=\sqrt{\beta \xi_{1} \gamma_{1}\left(D_{1} \pm d_{1}\right)+\beta \xi_{2} \gamma_{2}\left(D_{2} \pm d_{2}\right)} \zeta_{ \pm}(t),
$$

where $\beta \xi_{i}$ represents the fraction of the spontaneously emitted photons that goes into the zone $i$ of the lasing mode, $\zeta_{ \pm}$are two independent complex Gaussian random numbers, with zero mean $\left\langle\zeta_{ \pm}(t)\right\rangle=0$ and correlation $\left\langle\zeta_{ \pm}(t) \zeta_{ \pm}^{*}\left(t^{\prime}\right)\right\rangle=2 \delta\left(t-t^{\prime}\right)$. The coupling term $\Theta_{S, M}$, sketched in Fig. 1, takes the form $\Theta_{S}=\Gamma\left(F_{ \pm M}+H(\omega) F_{ \pm S}\right)$, $\Gamma$ being the injection attenuation and $\Theta_{M}=0$.

First we briefly consider one of the dynamic regimes exhibited by any of the two systems, without coupling $\Theta_{S, M}=0$. A previous study ${ }^{9}$ indicate that a region of chaotic behavior is present in the phase space $\mu_{1} / \mu_{t h 1}$ vs birefringence. A detailed analysis shows that in that region the electric field exhibits a chaotic behavior in both polarization and total intensity, with at least 2 positive Lyapunov exponents.
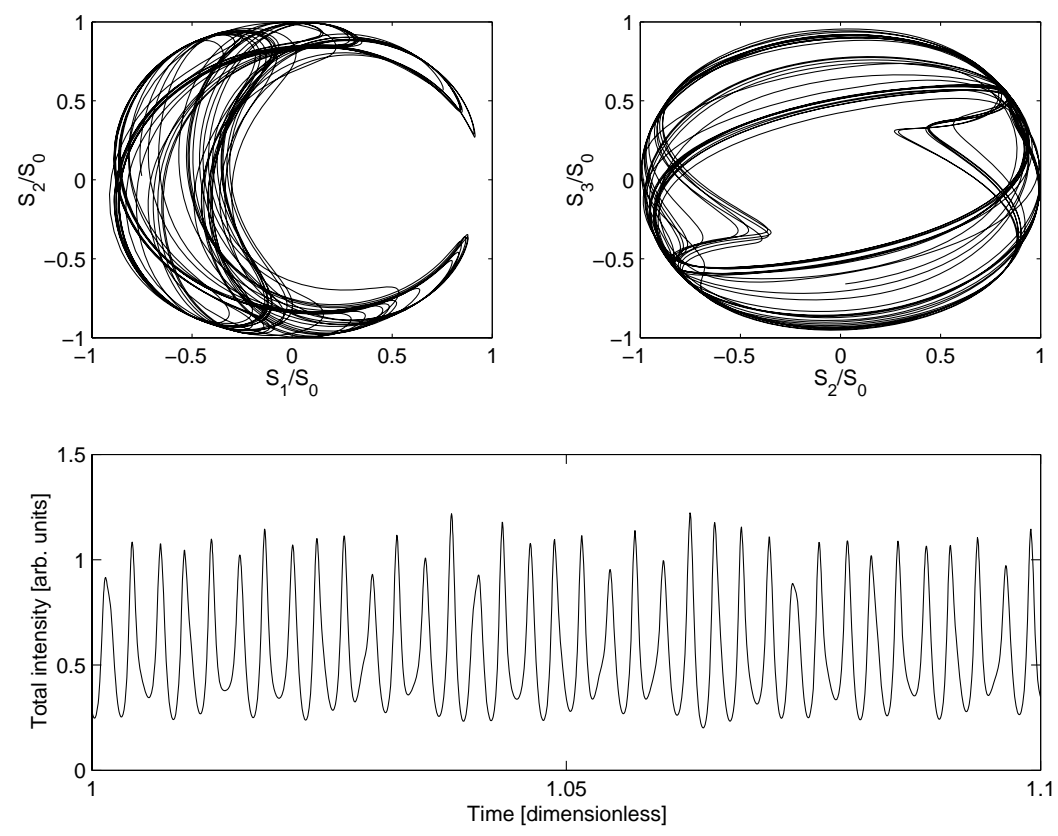

Figure 2. Vectorial chaos: chaotic attractor in the Poincaré sphere and chaotic evolution of the total intensity. All the numerical studies have been performed with the following choice of parameters: $\mu_{2}=-32, \gamma_{1}=1.09 \times 10^{-3}$, $\gamma_{2}=1.13 \times 10^{-3}, \alpha=3, \kappa=390 \mathrm{~ns}^{-1}, c_{12}=2.84 \times 10^{-2}, c_{21}=1.91, \gamma_{s 1}=0.25, \gamma_{s 2}=0.25, a_{2}=8.7$. This figure has been obtained by setting $\mu_{1} / \mu_{1 t h}=3, \gamma_{p}=20 n s^{-1}, \beta=0$. 
Fig. 2 shows two projections of the Poincaré sphere, through the Stokes parameters $S_{i}$, where $i=0,1,2$ and 3 , and the time trace of the total intensity. The electric field is therefore a vectorial magnitude changing its polarization state over a chaotic attractor in the Poincaré sphere. At the same time, the amplitude of the electric field vector is chaotically fluctuating. For simplicity, we assume that $M$ and $S$ share the same nominal values of all parameters (twin systems). As it is well known, even twin systems would follow completely different trajectories if they start from slightly different initial conditions and are isolated from each other. If we set the phase in the feedback loop of $S$ to $\phi=\pi$ and increase $\Gamma$, we find that after a certain value of $\Gamma=\Gamma_{c}$ the trajectories of the $M$ and $S$ systems get closer and closer, until they synchronize. By definition, ${ }^{11}$ synchronization means that $\left|\vec{F}_{M}-\vec{F}_{S}\right| \rightarrow 0$ as $t \rightarrow \infty$, in practice the output vector fields $\vec{F}_{M}$ and $\vec{F}_{S}$ of $M$ and $S$ are expected to become virtually coincident after a sufficiently long time of interaction, if $\Gamma \geq \Gamma_{c}$. To quantitatively express the degree of synchronization we generalize the scalar mean relative error ${ }^{11}$ to a vectorial magnitude, written as $\vec{\sigma}=\left(\sigma_{+}, \sigma_{-}\right)$for their circular components, where

$$
\sigma_{ \pm}=\frac{<F_{ \pm M}-F_{ \pm S}>}{<F_{ \pm M}>}
$$

where $\langle\cdot\rangle$ means temporal average. In Fig. 3 we show the transition from a unsynchronized state $\left(\sigma_{ \pm} \sim 1\right)$ to a synchronized state $\left(\sigma_{ \pm} \ll 1\right)$, in absence of noise $(\beta=0)$.

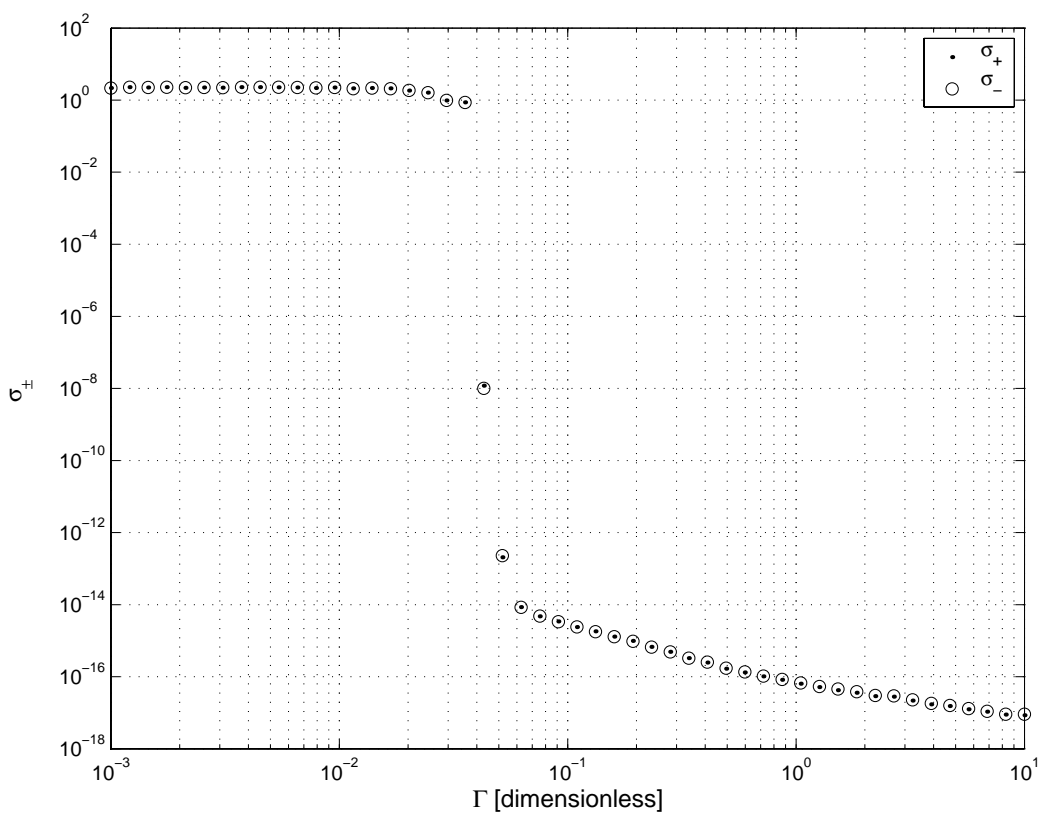

Figure 3. Transition from an unsynchronized $\left(\sigma_{ \pm} \sim 1\right)$ state to synchronization increasing the feedback stiffness $\Gamma$. Synchronization takes place at $\Gamma \sim 0.05$.

A high quality synchronization is thus reachable under ideal conditions. In agreement with, ${ }^{11,12}$ the introduction of spontaneous emission noise does not affect appreciably the synchronization. In Fig. 4 we show the good synchronization achieved in total intensity, and polarization (Fig. 5), for a particular value of $\Gamma=0.9>\Gamma_{c}$, under noisy conditions $\left(\beta=5 \times 10^{-7}\right)$. In particular, in Fig. 5 we show the Stokes parameters differences $\Sigma_{i}$ defined as follows

$$
\Sigma_{i}=\log _{10}\left|\frac{S_{i M}}{S_{0 M}}-\frac{S_{i S}}{S_{0 S}}\right|
$$

where $S_{i M, S}(i=0,1,2$ and 3$)$ are the Stokes parameter describing the polarization state of the master $(M)$ and slave $(S)$ systems. By setting the phase $\phi=\pi$ we impose a negative feedback condition, thus introducing a dissipative term in $F_{ \pm S}$ in Eqs. (2). It is known ${ }^{14}$ that this kind of term has a stabilizing effect, because it lowers the Lyapunov exponents. From a practical point of view, the accuracy needed to fulfil the condition $\phi=\pi$, was found to be critical. 
However, the accuracy level to be met is of the same order as in coherent detection or interferometry. For example, the difference $\vec{F}_{M}-\vec{F}_{S}$ requires coherent field superposition (with $\pi$ phase shift) at a beam combiner; a suitable active control of the path-length must be introduced, since, for efficient synchronization, the residual phase error must not exceed a few degrees, as we found in numerical simulations shown in Fig. 6 .

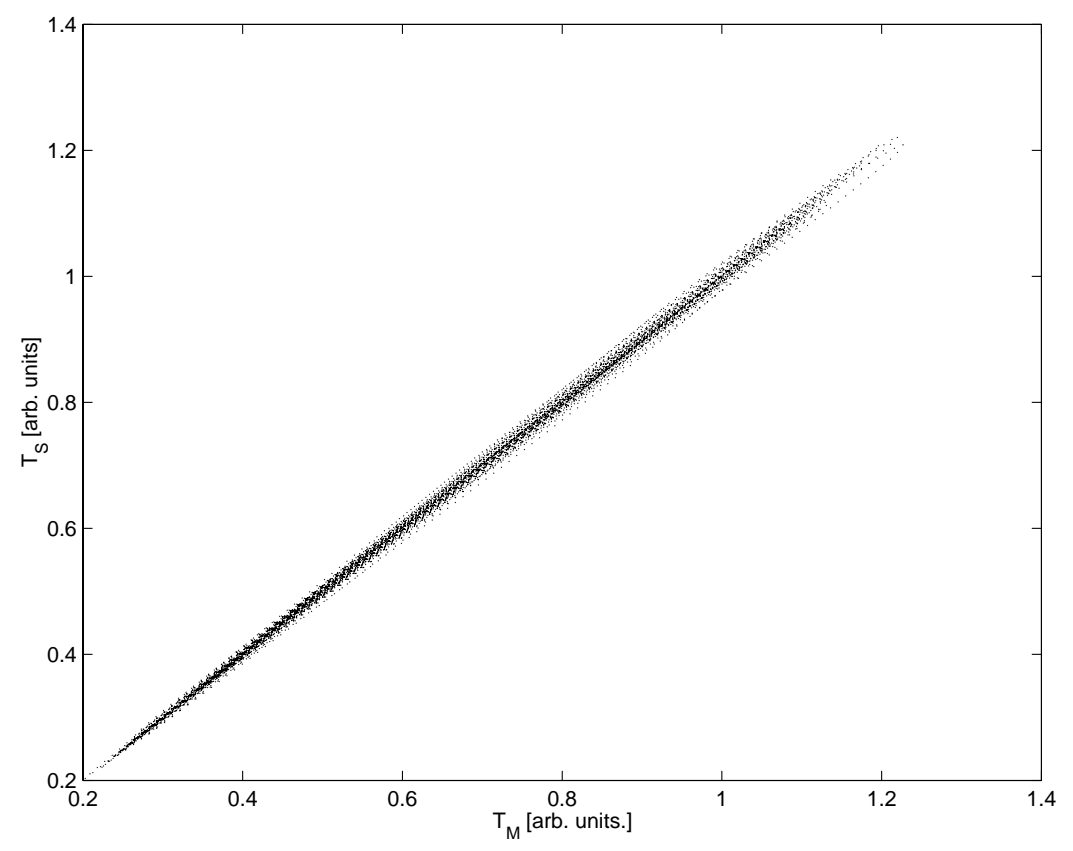

Figure 4. Return plot of the total intensities.
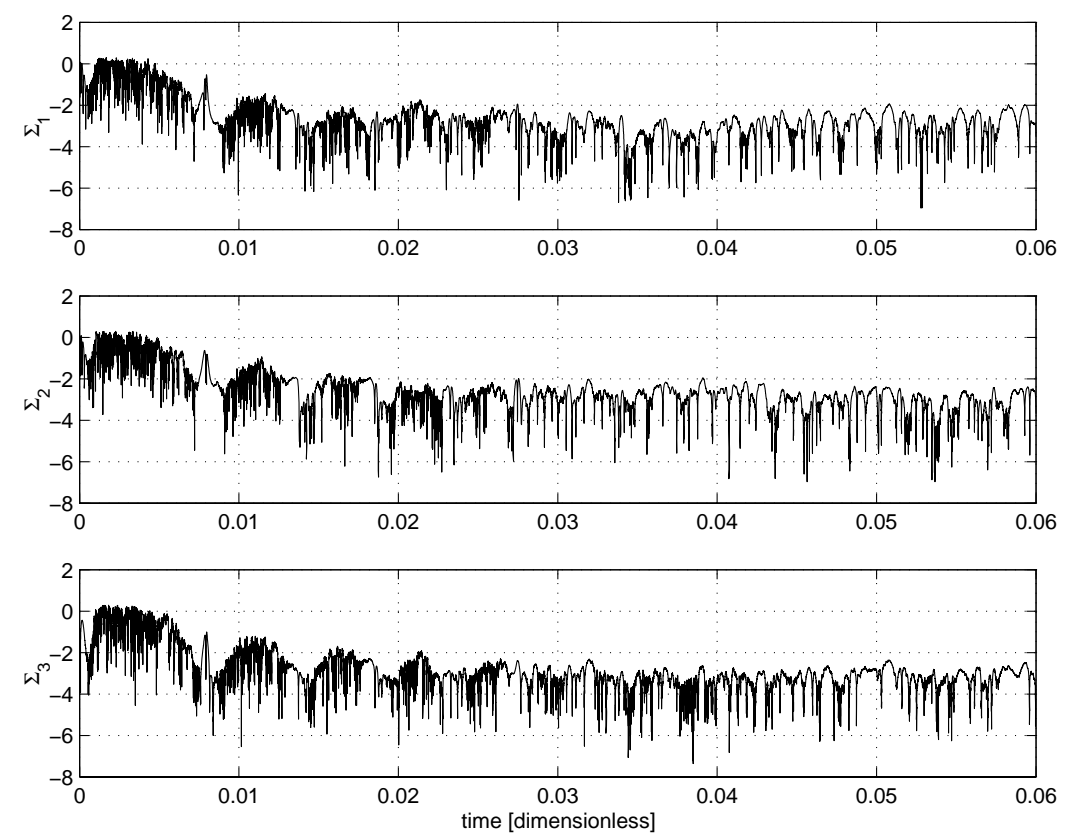

Figure 5. Synchronization of the Stokes parameters. 


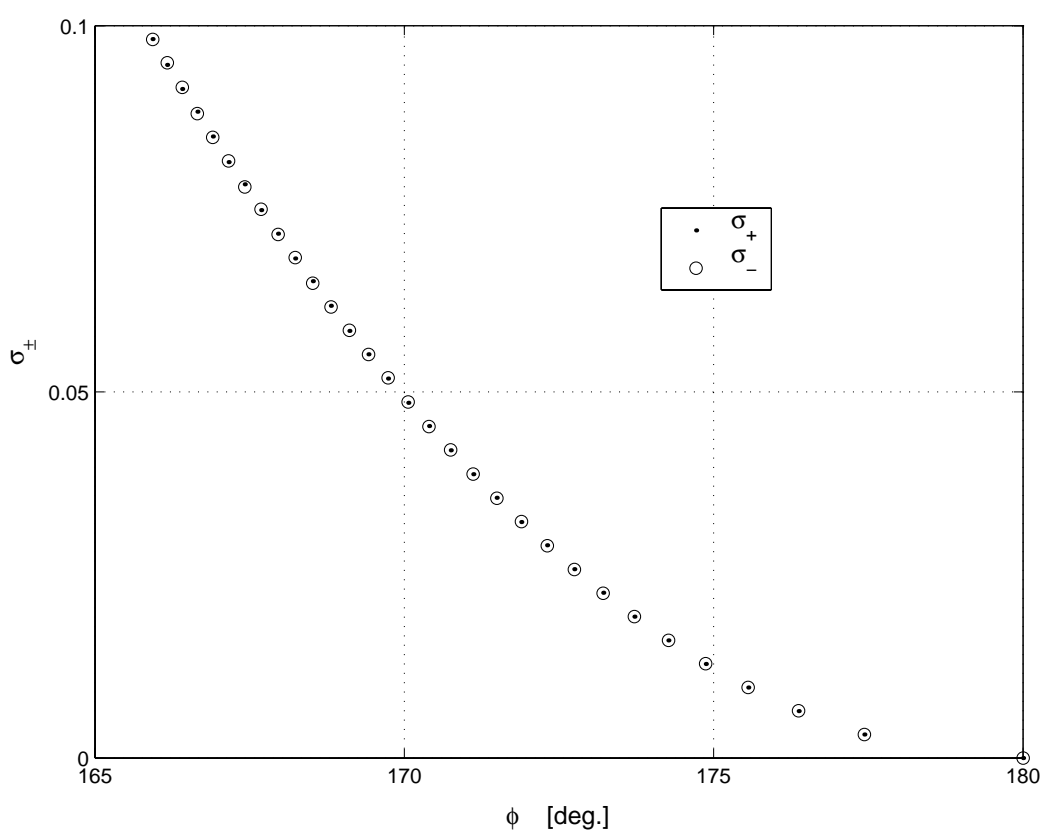

Figure 6. Impact of a deviation from the optimum feedback phase condition $(\phi=\pi)$ on the synchronization error.

\section{CSK ENCODING}

In this section we show how the synchronization method, described in the previous section, can be exploited in a secure communication scheme. The proposed structure (shown in fig. 7) consists in a double-contact VCSEL, where the current $I_{1}$ represents the usual pump, while the current $I_{2}$ represents an independent control of the carrier density in the absorber.

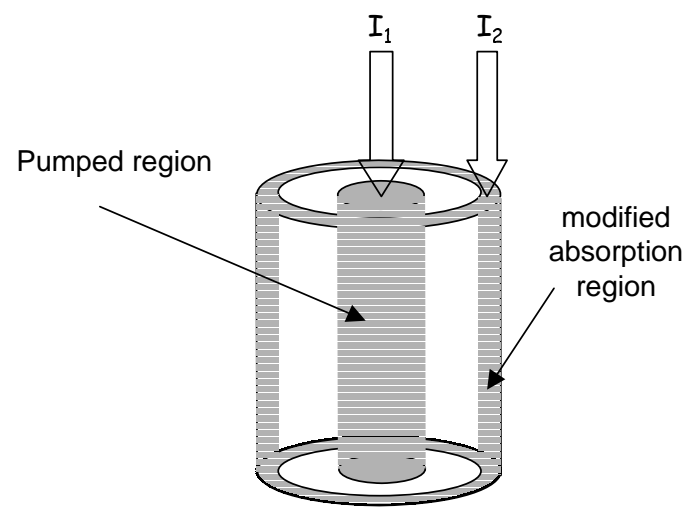

Figure 7. Schematic representation of a double-contact VCSEL. The properties of the saturable absorber are changed through the control current $I_{2}$.

The effect of $I_{2}$ is to modify the effective absorber current $\mu_{2}$ in Eq. (4). This structure is analogous of the wellknown two contacts EELs. By exploiting the added degree of freedom, a message can be encoded into in the system in the following way: $I_{1}-I_{2}=s(t)$, while the total current supplied to the device can be left constant: $I_{1}+I_{2}=I_{\text {tot }}$. The chaotic evolution of the total intensity is unaffected by this kind of modulation, while a variation of the total 
current $I_{t o t}$ would affect the mean value and fluctuation extent of the chaos in total intensity, leading to an easily recognizable modulation. The demodulation scheme is a standard On-Off Chaos Shift Keying (OOCSK) ${ }^{11,12,16}$ : one double-contact VCSEL, biased in the "0" ("1") configuration is set as a Slave in a CCS. The Slave synchronizes (de-synchronizes) to the received signal when a bit " 0 " ("1") is received. The maximum bit-rate is limited by the synchronization time. In the CCS, ${ }^{15}$ the synchronization time linearly decreases with the feedback stiffness $\Gamma$.
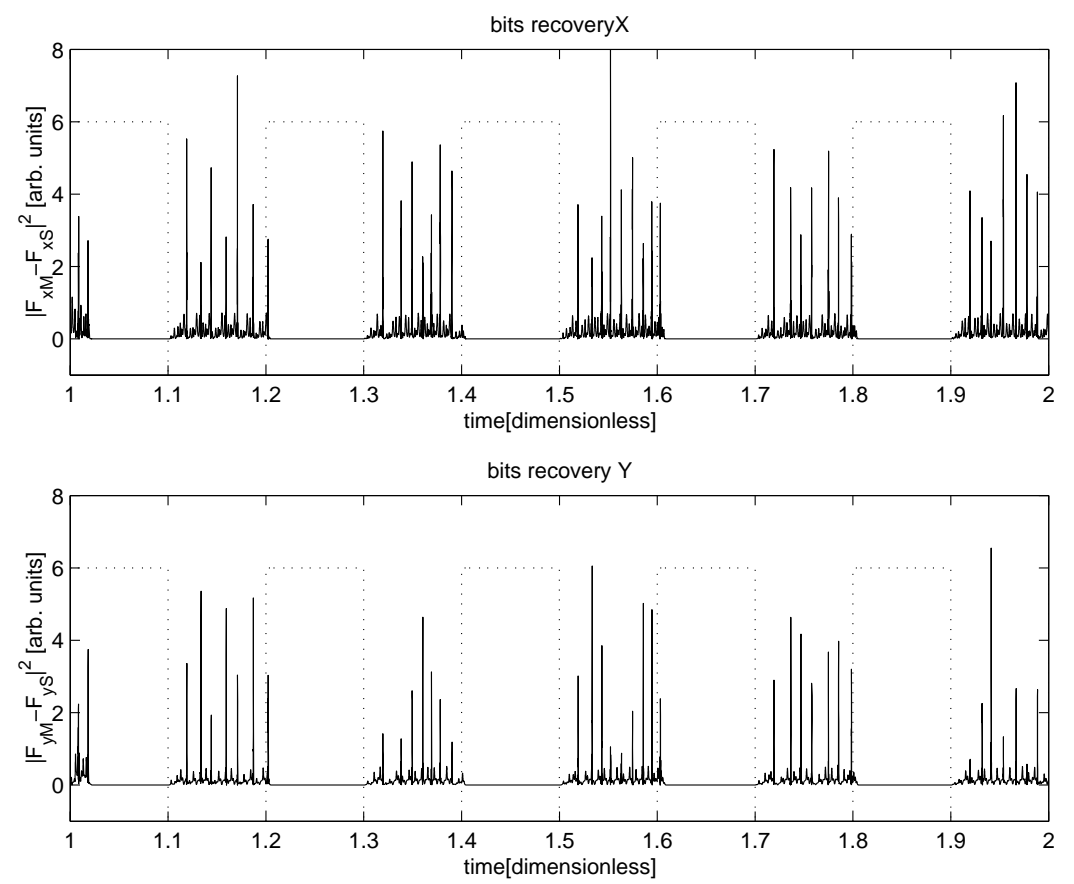

Figure 8. Message recovery. Two channels associated to two orthogonal polarization components synchronize when a "0" is sent. The current difference modulation signal is $\mu_{1}-\mu_{2}=80$ for "0" and $\mu_{1}-\mu_{2}=60$ for "1" in the Master system. The slave system is set to $\mu_{1}-\mu_{2}=80$, that is the "0" bit.

In Fig. 8 we show the bits reconstruction at the receiver, for two linear components of the electric field $\vec{F}_{S}$. The vectorial nature of the synchronized chaos is exploited to lower the probability error. Assuming that the two channels are independent, the probability error can be written as $P(E)=p_{x}(E) p_{y}(E)$. Due to the symmetry of the problem, we found that $p_{x}=p_{y}$. Moreover $p_{x(y)}$ was found to be of the same order of the probability error encountered in a EELs based CSK system. ${ }^{11}$ The probability error is thus lowered from $p_{x(y)}$ to $p_{x(y)}^{2}$. Alternatively, one can exploit the vectorial nature of the chaotic carrier to enhance the transmission capacity, implementing a two channels chaotic masking. In this scheme one superimposes two independent signals with orthogonal polarization to the vectorial carrier. At the receiver, once the synchronization is achieved through the CCS, the two signals can be recovered by doing chaos filtering.

\section{CONCLUSIONS}

In conclusion, we have discussed the synchronization properties of the chaos generated by two VCSELs in presence of a saturable absorber. The vectorial nature of the chaotic field requires a continuous control scheme to achieve a highquality synchronization between the lasers. We proposed a novel encoding scheme, exploiting a two contact VCSEL structure, which allows an easy and direct modulation of the transmitter. The added degree of freedom constituted by the chaos in polarization permits to decrease the probability error (or enhance the transmission capacity). 


\section{Acknowledgements}

This work has been funded by the European Commission through the VISTA HP-TRN, Marie Curie IF MCFI-200000617, OCCULT project, IST-2000-29683 and the Spanish MCyT under projects TIC99-0645-C05-02, BFM20001108, MCyT SINFIBIOS BFM 2001-0341-C02-01.

\section{REFERENCES}

1. L. M. Pecora and L. Carrol, "Synchronization in chaotic circuits," IEEE Trans. Circuit Syst., vol. 38, p. 453, 1991.

2. J. Mork, B. Tromborg, and J. Mark, "Chaos in semiconductor lasers with optical feedback: Theory and experiments," IEEE J. Quantum Electron., vol. 28, p. 93, 1992.

3. T. Sugawara, M. Tachikawa, T. Tsukamoto, and T. Shimizu, "Observation of synchronization in laser chaos," Phys. Rev. Lett., vol. 72, p. 3602, 1994.

4. L.M.Pecora and L. Carrol, "Synchronization in chaotic systems," Phys. Rev. Lett., vol. 64, p. 821, 1990.

5. C. R. Mirasso, P. Colet and P. Garcia-Fernandez, "Synchronization of chaotic semiconductor lasers: Application to encoded communications," IEEE Photon. Technol. Lett vol. 8, p. 299, 1996.

6. T. Heil, I. Fisher, W. Elsässer, J. Mulet and C. R. Mirasso, "Chaos synchronization and spontaneous symmetrybreaking in simmetrically delay-coupled semioconductor lasers," Phys. Rev. Lett., vol. 86, p. 795, 2001.

7. R. Roy and K. S. Thornburg Jr., "Experimental synchronization of chaotic lasers," Phys. Rev. Lett., vol. 72, p. R7359, 1994.

8. K. J. Ebeling in Semiconductor Quantum Optoelectronics, edited by A. Miller, M.Ebrahimzadeh and D.M. Finlaynson (Institute of Physics, Bristol, 1999), p.339.

9. A. Scirè, J. Mulet, C. R. Mirasso, and M. San Miguel. "Total intensity and polarization self-pulsations in VCSELs," to appear in Opt. Lett., (2002).

10. A. Scirè, J. Mulet, C. R. Mirasso, and M. San Miguel. "Total intensity and polarization self-pulsations in VCSELs," Proc. of SPIE 2002, Paper OE-4646-26.

11. V. Annovazzi-Lodi, S. Donati and A. Scirè, "Synchronization of chaotic injected-laser systems and its application to optical cryptography," IEEE J. Quantum Electron. vol. 32, p. 953, 1996.

12. V. Annovazzi-Lodi, S. Donati and A. Scirè, "Synchronization of chaotic lasers by optical feedback for cryptographic applications," IEEE J. Quantum Electron. vol. 33, p. 953, 1997.

13. A. Scirè, "Non linear phenomena due to injection in semiconductor lasers," PhD dissertation, Universita' di Pavia, 1999.

14. M. S. Vieira, A. J. Lichtenberg, and M. A. Lieberman, "Synchronization of regular and chaotic systems," Phys. Rev. A vol. 46, p. R7359, 1992.

15. T. Kapitaniak, "Synchronization of chaos using continuous control," Phys. Rev. E vol. 50, p. $1642,1994$.

16. J. B. Cuenot, L. Larger, J. P. Goedgebuer, and W. T. Rhodes, "Chaos shift keying with an optoelectronic encryption system using chaos in wavelength," IEEE J. Quantum Electron. vol. 37, p. 849, 2001.

17. M. San Miguel, Q. Feng and J. V. Moloney, "Light-polarization dynamics in surface-emitting semiconductor lasers," Phys. Rev. A, vol. 52, p. 1728, (1995).

18. M. Yamada, "A Theoretical Analysis of Self-Sustained Pulsation Phenomena in Narrow-Stripe Semiconductor Lasers," IEEE J. Quantum Electron. vol. 29, p. 1330, 1993.

19. J. Mulet, C. R. Mirasso and M. San Miguel, "Polarization resolved intensity noise in vertical-cavity surface -emitting lasers," Phys. Rev. A, vol. 64, p. 023817, 2001. 